\title{
Pre-disease pregnancy complications and systemic sclerosis: pathogenic or pre-clinical?
}

\author{
Eliza Chakravarty* \\ See related research by van Wyk et al., http://arthritis-research.com/content/13/6/R183
}

\begin{abstract}
The fetal microchimerism theory for the pathogenesis of systemic sclerosis (SSc) has compelling biologic support, including the female predominance of the disease, the mean age of onset after childbearing years, similarities between diffuse cutaneous SSc and graft-versus-host disease, as well as the detection of microchimeric cells in peripheral blood and skin of SSc patients. The previous issue of Arthritis Research and Therapy presents findings of a positive association between pregnancy complications and future diagnosis of SSc in parous women. Before interpreting the results of this epidemiologic study as support for fetal microchimerism, however, other theories for the observed associations must be considered.
\end{abstract}

Akin to many autoimmune diseases with a strong female predominance, relationships between pre-disease pregnancies and subsequent development of systemic sclerosis (SSc) have been the source of a great deal of study, often with conflicting results.

Fetal microchimerism - the bidirectional transfer of cells between mother and fetus during gestation and delivery, followed by perpetuation of small amounts of foreign cells or DNA in the host for years or decades, potentially leading to a graft-versus-host-like effect - has been implicated in the pathogenesis of SSc based upon epidemiologic and immunological studies. While the fetal microchimerism theory of SSc carries compelling biologic plausibility given the clinical resemblance of diffuse cutaneous SSc and graft-versus-host disease, as well as the increased presence of fetal cells in women with SSc, purely epidemiologic studies without translational, immunological, or cellular correlates cannot directly support a causal association.

*Correspondence: chakravartye@omrf.org

Department of Arthritis and Clinical Immunology, Oklahoma Medical Research Foundation, 825 NE $13^{\text {th }}$ Street, Oklahoma City, OK 73104, USA
The current study by van Wyk and colleagues is an excellent example of a well-performed epidemiologic study identifying a positive association between pregnancy complications (hypertensive disorders of pregnancy (HTN) and intrauterine growth restriction (IUGR)) prior to diagnosis and the future development of SSc [1]. The authors conducted a case-control study of parous women with SSc compared with healthy women. Reproductive history was obtained through questionnaires. The mean age at enrollment was 57 years, approximately 30 years after the first pregnancy for both groups. Results showed a statistically significant increase of HTN as well as IUGR among women who later developed SSc. Notably, the rates of HTN and IUGR among women in this study who were later diagnosed with SSc were not demonstrably different from the rates reported for women with preexisting SSc (22.9\% HTN [2] and 5 to $20 \%$ IUGR [2,3]).

Despite the merits of this study, it remains difficult to interpret the results as directly supportive of fetal microchimerism. Certainly, many pregnancy complications increase maternal-fetal cellular trafficking. However, maternal-fetal cellular transfer is seen in almost all pregnancies, and is increased in other pregnancy disorders not reported in this study including preterm labor and pregnancy termination [4]. Other modes of microchimerism, including blood transfusions, are not assessed in the study. What perhaps may be more relevant to understanding the relationship between prior pregnancy complications and future diagnosis of SSc is to uncover whether fetal cells are perpetuated in maternal blood and tissues and to identify activation markers on fetal cells that may contribute to disease-related inflammation and fibrosis. Mere transfer of fetal cells is probably not enough; and mechanisms of perpetuation and activation of fetal cells in maternal tissue may be entirely independent of the amount of cellular transfer during pregnancy [5].

When thinking about the pathogenesis of SSc, it has becoming increasingly clear that combining both limited and diffuse forms together may obscure important associations - the clinical manifestations, autoantibody profile and natural history are quite different between the two 
subtypes. Limited cutaneous SSc is characterized by a more prominent vasculopathy with less cutaneous fibrosis, is associated with anti-centromere antibodies, and generally has a prolonged clinical course prior to diagnosis. In contrast, diffuse cutaneous SSc more closely resembles graft-versus-host disease, has more prominent cutaneous and organ fibrosis, is associated with antitopoisomerase I antibodies, and has a shorter period between symptom onset and diagnosis [6]. Fetal microchimerism may therefore not play the same role in the development of different subsets of SSc. Indeed, a recent study of women with SSc found that male microchimeric cells were contained in different maternal peripheral blood compartments in limited cutaneous SSc (whole blood) compared with diffuse cutaneous SSc (peripheral blood mononuclear cells) [7]. This study also found associations between maternal-fetal HLA-DRB1 compatibility only in the limited cutaneous SSc subset compared with controls: no associations were seen among women with diffuse cutaneous SSc; and no significant differences were seen when diffuse cutaneous SSc patients and limited cutaneous SSc patients were combined [7]. van Wyk and colleagues' study did not separate women with limited and diffuse cutaneous SSc, raising the potential that even stronger associations may be seen between prior pregnancy events and future development of disease in a particular subset that may either strengthen or diminish the possibility of fetal microchimerism as a potential etiologic factor.

Even purely epidemiologic studies of SSc have yielded conflicting data regarding associations between development of disease and previous pregnancies. Some studies find an independent association between parity and later development of SSc [8], while others find the greatest risk of SSc to be in nulliparous women [9]. Other studies have found that nulliparous women developed SSc approximately 10 years earlier than parous women (32 vs. 45.7 years) and were more likely to have diffuse cutaneous disease with internal organ involvement [10].

Associations between previous pregnancies and future development of SSc thus remain puzzling. While fetal microchimerism may indeed play a role in the development of some cases of SSc, alternative biologic theories to explain the associations seen in van Wyk and colleagues' study must also be considered.

An entirely separate theory for these findings posits that pregnancy complications, particularly vascular complications including hypertension and IUGR, may be a manifestation of very early, pre-clinical manifestations of SSc in otherwise healthy women. The data provided in this study showing a prolonged time between first pregnancy and diagnosis of SSc of nearly 30 years may argue against this theory; however, the age of onset of Raynaud's phenomenon or the first non-Raynaud's manifestation is not reported in the study, nor is the age of SSc diagnosis ascertained for $40 \%$ of respondents. Similarly, maternal age at last pregnancy is not reported, so the time from final fetal-to-maternal trafficking to onset of symptoms cannot be estimated. It is well known that, particularly for limited cutaneous SSc, diagnosis occurs years to decades after onset of Raynaud's phenomenon. The increased blood volume and other cardiopulmonary changes of pregnancy may possibly also lead to vascular compromise in pre-clinical phases of systemic vasculopathy that would otherwise remain asymptomatic for many years.

\section{Abbreviations}

HTN, hypertensive disorders of pregnancy; IUGR, intrauterine growth restriction; SSc, systemic sclerosis.

\section{Competing interests}

The author declares that she has no competing interests.

Published: 6 February 2012

\section{References}

1. van Wyk L, van der Marel J, Schuerwegh AJM, Schouffoer AA, Voskuyl AE, Huizinga TWJ, Bianchi DW, Scherjon SA: Increased incidence of pregnancy complications in women who later develop scleroderma: a case control study. Arthritis Res Ther 2011, 13:R183.

2. Chung L, Flyckt RLR, Colon I, Shah AA, Druzin M, Chakravarty EF: Outcome of pregnancies complicated by systemic sclerosis and mixed connective tissue disease. Lupus 2006, 15:595-599.

3. Chakravarty EF, Khanna D, Chung L: Pregnancy outcomes in systemic sclerosis, primary pulmonary hypertension, and sickle cell disease. Obstet Gynecol 2008, 111:927-934.

4. Artlett CM: Pathophysiology of fetal microchimeric cells. Clin Chim Acta 2005, 360:1-8.

5. Jimenez SA, Artlett CM: Microchimerism and systemic sclerosis. Curr Opin Rheumatol 2004, 17:86-90.

6. Nikpour M, Stevens WM, Herrick AL, Proudman SM: Epidemiology of systemic sclerosis. Best Pract Res Clin Rheumatol 2010, 24:857-869.

7. Rak JM, Pagni PP, Tiev K, Allanore Y, Farge D, Harle JR, Launay D, Hachulla E, Didelot R, Cabane J, Kahan A, Martin M, Granel B, Roudier J, Lambert NC: Male microchimerism and HLA compatibility in French women with scleroderma: a different profile in limited and diffuse subset. Rheumatology 2009, 48:363-366.

8. Cockrill T, del Junco D, Arnett FC, Assassi S, Tan FK, McNeaney T, Fischbach M, Perry M, Mayes MD: Separate influences of birth order and gravidity/parity on the development of systemic sclerosis. Arthritis Care Res 2010, 62:418-424.

9. Lambe M, Bjornadal L, Neregard P, Nyren O, Cooper GS: Childbearing and the risk of scleroderma: a population-based study in Sweden. Am J Epidemiol 2004, 159:162-166.

10. Artlett CM, Rasheed M, Ruzzo-Steiglitz KE, Saways HHB, Jimenez SA: Influence of prior pregnancies on disease course and cause of death in systemic sclerosis. Ann Rheum Dis 2002, 61:346-350.

doi:10.1186/ar3686

Cite this article as: Chakravarty E: Pre-disease pregnancy complications and systemic sclerosis: pathogenic or pre-clinical? Arthritis Research \& Therapy 2012, 14:102. 